\title{
Construction and Performance of Large-Area Triple-GEM Prototypes for Future Upgrades of the CMS Forward Muon System
}

\author{
M. Tytgat*, Member, IEEE, A. Marinov, N. Zaganidis, Y. Ban, J. Cai, H. Teng, A. Mohapatra, T. Moulik, \\ M. Abbrescia, A. Colaleo, G. de Robertis, F. Loddo, M. Maggi, S. Nuzzo, S. A. Tupputi, L. Benussi, S. Bianco, \\ S. Colafranceschi, D. Piccolo, G. Raffone, G. Saviano, M.G. Bagliesi, R. Cecchi, G. Magazzu, E. Oliveri, \\ N. Turini, T. Fruboes, D. Abbaneo, C. Armagnaud, P. Aspell, S. Bally, U. Berzano, J. Bos, K. Bunkowski, \\ J. P. Chatelain, J. Christiansen, A. Conde Garcia, E. David, R. De Oliveira, S. Duarte Pinto, S. Ferry, F. Formenti, \\ L. Franconi, A. Marchioro, K. Mehta, J. Merlin, M. V. Nemallapudi, H. Postema, A. Rodrigues, L. Ropelewski, \\ A. Sharma, Senior Member, IEEE, N. Smilkjovic, M. Villa, M. Zientek, A. Gutierrez, P. E. Karchin, K. Gnanvo, \\ M. Hohlmann, Member, IEEE, M. J. Staib
}

\begin{abstract}
At present, part of the forward RPC muon system of the CMS detector at the CERN LHC remains uninstrumented in the high- $\eta$ region. An international collaboration is investigating the possibility of covering the $1.6<|\eta|<2.4$ region of the muon endcaps with large-area triple-GEM detectors. Given their good spatial resolution, high rate capability, and radiation hardness, these micro-pattern gas detectors are an appealing option for simultaneously enhancing muon tracking and triggering capabilities in a future upgrade of the CMS detector. A general overview of this feasibility study will be presented. The design and construction of small $\left(10 \times 10 \mathbf{c m}^{2}\right)$ and full-size trapezoidal $\left(1 \times 0.5 \mathbf{m}^{2}\right)$ triple-GEM prototypes will be described. During detector assembly, different techniques for stretching the GEM foils were tested. Results from measurements with $x$-rays and from test beam campaigns at the CERN SPS will be shown for the small and large prototypes. Preliminary simulation studies on the expected muon reconstruction and trigger performances of this proposed upgraded muon system will be reported.
\end{abstract}

\section{INTRODUCTION}

Manuscript received November 15, 2011

A. Marinov, M. Tytgat, N. Zaganidis are with the Dept. of Physics and Astronomy, Ghent University, Gent, Belgium

Y. Ban, J. Cai, H. Teng are with Peking University, Beijing, China

A. Mohapatra, T. Moulik are with NISER, Bhubaneswar, India

M. Abbrescia, A. Colaleo, G. de Robertis, F. Loddo, M. Maggi, S. Nuzzo, S. A. Tupputi are with Politecnico di Bari, Università di Bari, and INFN Sezione di Bari, Bari, Italy

L. Benussi, S. Bianco, S. Colafranceschi, D. Piccolo, G. Raffone, G. Saviano are with the Labortori Nazionali di Frascati INFN, Frascati, Italy

M.G. Bagliesi, R. Cecchi, G. Magazzu, E. Oliveri, N. Turini are with INFN Sezione di Pisa, Pisa, Italy

T. Fruboes is with Warsaw University, Warsaw, Poland

D. Abbaneo, C. Armagnaud, P. Aspell, S. Bally, U. Berzano, J. Bos, K. Bunkowski, J. P. Chatelain, J. Christiansen, A. Conde Garcia, E. David, R. De Oliveira, S. Duarte Pinto, S. Ferry, F. Formenti, L. Franconi, A. Marchioro, K. Mehta, J. Merlin, M.V. Nemallapudi, H. Postema, A. Rodrigues, L. Ropelewski, A. Sharma, N. Smilkjovic, M. Villa, M. Zientek are with the Physics Department, CERN, Geneva, Switzerland

A. Gutierrez, P. E. Karchin are with the Dept. of Physics and Astronomy, Wayne State University, Detroit, MI, USA

K. Gnanvo, M. Hohlmann, M. J. Staib are with the Dept. of Physics and Space Sciences, Florida Institute of Technology, Melbourne, FL, USA

* Corresponding author, michael.tytgat@cern.ch

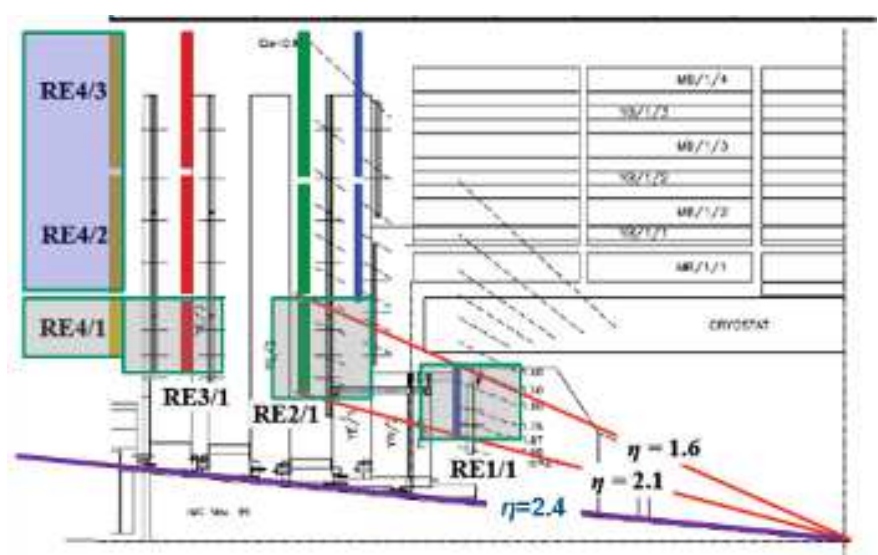

Fig. 1. One quarter of the CMS muon system. The colored detectors are the RPCs in the endcap system. RPCs (REi/1 and RE4/2-3) enclosed in boxes have yet to be installed.

T HE Compact Muon Solenoid (CMS) experiment [1] has been collecting data successfully since the start of the first Large Hadron Collider (LHC) physics run in 2009. During two future long shutdown periods of the accelerator, the CMS Collaboration intends to upgrade several subsystems of its detector [2]. In particular, the muon system as depicted in Fig. 1 will be extended by completing rings 2 and 3 in the fourth station in both endcaps to ensure efficient muon triggering and reconstruction in that region as the LHC instantaineous luminosity continues to increase. During the first Long Shutdown presently scheduled for 2013-2014, this fourth endcap station will be equipped with Resistive Plate Chambers (RPCs) up to $|\eta|<1.6$. For the latter subsystem, which is a dedicated CMS muon trigger detector, the very forward region beyond $|\eta|=1.6$ will remain empty and could in principle be instrumented up to $|\eta|=2.4$ as is already the case for the Cathode Strip Chamber (CSC) system that serves as muon tracker in the endcap region. However, the present design of the endcap RPCs, made of a double Bakelite gas gap and operating in avalanche mode, is not expected to be suitable for 
the particle rates amounting to several tens of $\mathrm{kHz} / \mathrm{cm}^{2}$ in the scenario of an LHC luminosity going up to $10^{34-35} \mathrm{~cm}^{-2} \mathrm{~s}^{-1}$.

Here, we report on an ongoing feasibility study to insert Gas Electron Multiplier (GEM) detectors in the vacant space of the RPC endcap system beyond $|\eta|=1.6$. In general, GEM detectors feature excellent spatial $(\sim 100 \mu \mathrm{m})$ and timing ( $\sim 5 \mathrm{~ns})$ resolution and are proven to be able to withstand particle rates up to $10 \mathrm{MHz} / \mathrm{cm}^{2}$. Furthermore, in the specific case of CMS, the use of such detector technology could in principle allow the combination of muon triggering and tracking capabilities into one single subsystem.

Generally desired for a trigger/tracker detector in the CMS high $\eta$ region are a time resolution better than $5 \mathrm{~ns}$, a spatial resolution in the range of $200-400 \mu \mathrm{m}$ and an overall detector efficiency exceeding $97 \%$.

\section{Studies with Small Prototypes}

In the course of this project that was initiated in 2009, several different types of small triple-GEM prototypes were produced and then studied using x-rays in the RD51 [3] lab of the CERN Detector Technology Group (DT) and/or particle beams at the CERN SPS. Table [ specifies the small detectors that were produced so far along with their main construction parameters. The first three small triple-GEMs listed in the table were extensively tested during 2009-2010 using the $150 \mathrm{GeV}$ pion/muon beam of the CERN SPS H4 beam line. The main test results are summarized below; more details can be found in [4].

The "Timing GEM" was mainly used to study the time resolution that could be obtained with such detectors, as function of the used gas mixture, and the drift and induction fields. A time resolution of $4 \mathrm{~ns}$ could be reached with an $\mathrm{Ar} / \mathrm{CO}_{2} / \mathrm{CF}_{4} 45 / 40 / 15$ gas mixture and a 3/1/2/1 mm (drift, tranf.1, transf.2, ind.) gap size configuration, which meets the requirement for the CMS muon triggering system.

Adopting a geometry for the GEMs similar to the RPCs in the CMS endcap disks, the smallest active GEM detector area needed by CMS is of the order of $50 \times 100 \mathrm{~cm}^{2}$. For such sizes, the standard double-mask technique to produce the GEM foils is not ideal as it suffers from alignment problems of the two masks on either side of the foils during the photolitographic hole etching process. The single-mask technique [5] overcomes this problem and was used to produce the "Single-Mask GEM" prototype. The performance of the single-mask GEM was quite similar to our "Timing GEM". An efficiency up to $98 \%$ was measured, albeit for a slightly higher gain than for the double-mask GEM. Nevertheless, the single-mask technique appears quite mature and was chosen for the production of our large prototypes.

Depending on how many endcap disks would be instrumented with GEMs, the number of detectors needed for CMS could amount to several 100s. For such quantities, the time and certainly the cost of the detector production becomes an issue. The most time-consuming and labor-intensive part of tripleGEM production is the foil stretching and the gluing of the spacer frames. To avoid these steps in the production process, two novel assembly procedures were tested as described below.
The "Honeycomb GEM" produced in 2010, was assembled using honeycomb structures as spacers in the detector gaps between the GEM foils, which avoids the need to stretch the foils. Although this prototype could be operated without any problems, very localized efficiency losses were observed at the position of the honeycomb structures. With honeycomb cell sizes of $(6 / 12 / 12 / 12) \mathrm{mm}$ or $(6 / 0 / 0 / 0) \mathrm{mm}$ in the (drift, transf.1, transf.2, ind.) gap, an overall detector efficiency of about $75 \%$ was obtained.

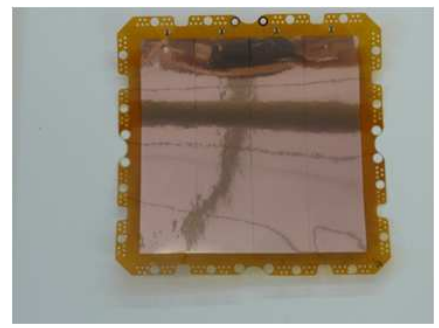

(a)

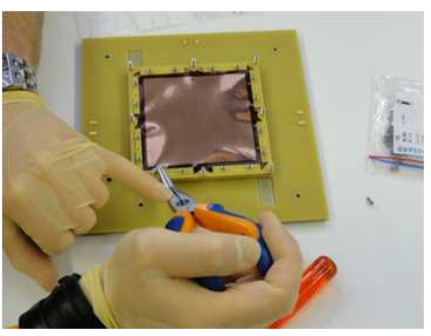

(c)

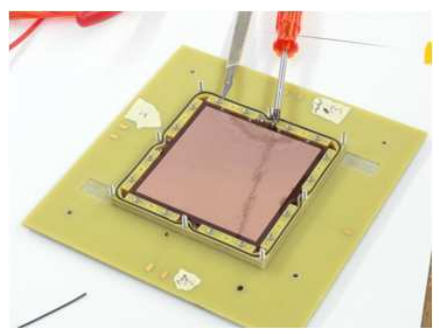

(e)

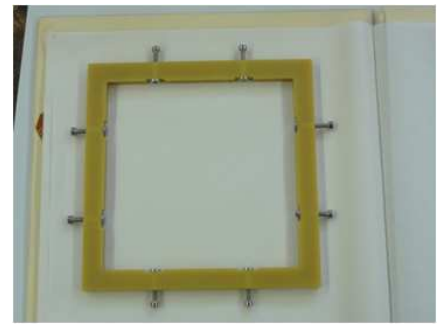

(b)

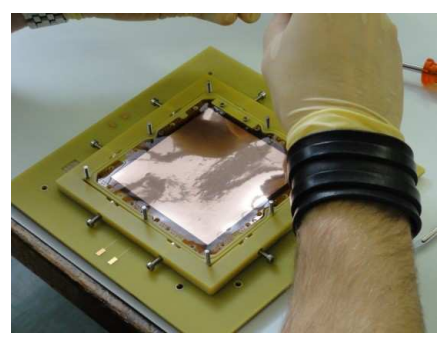

(d)

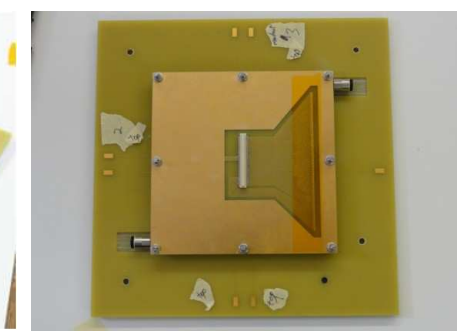

(f)
Fig. 2. The self-stretching triple-GEM assembly technique : (a) GEM foil before assembly; (b) external frame for foil stretching; (c) mounting the GEM foils; (d) stretching the foils; (e) stretched GEM; (f) completed detector including readout board.

An important development in 2011 was the introduction of another new GEM assembly technique, here referred to as the self-stretching technique. The procedure is demonstrated in Fig. 2] showing a few photographs taken during the assembly at CERN of the first $10 \times 10 \mathrm{~cm}^{2}$ triple-GEM, "CMS Proto III", prototype using this new technique. The GEM foils are produced with a pattern of holes in the edge on each of the four sides. The foils are then placed on a detector structure which has a set of alignment pins matching the hole pattern in the foil edges. Next, using a special external frame that is placed around the ensemble, the foils are mechanically stretched and then fixed with screws to the detector structure. In the end, once the foils have been completely stretched and fixed, the readout board can be mounted, closing the detector.

Clearly, compared to the standard assembly procedure, the 
TABLE I

OVERVIEW OF THE SMALL TRIPLE-GEM PROTOTYPES THAT WERE CONSTRUCTED AND STUDIED IN THIS PROJECT.

\begin{tabular}{|c|c|c|c|c|c|c|c|}
\hline Name & Mask Type & $\begin{array}{l}\text { Prod. } \\
\text { Tech. }\end{array}$ & $\begin{array}{l}\text { Active } \\
\text { Area } \\
\left(\mathrm{cm}^{2}\right)\end{array}$ & Readout & $\begin{array}{c}\text { Gap Sizes } \\
\text { (drift, transf. 1, transf. 2, ind.) } \\
(\mathrm{mm} / \mathrm{mm} / \mathrm{mm} / \mathrm{mm})\end{array}$ & \#strips & $\begin{array}{l}\text { Prod. } \\
\text { Site }\end{array}$ \\
\hline "Timing GEM" & double-mask & standard & $10 \times 10$ & $1 \mathrm{D}$ & $(3 / 2 / 2 / 2) \&(3 / 1 / 2 / 1)$ & 128 & CERN \\
\hline "Single-Mask GEM" & single-mask & standard & $10 \times 10$ & $2 \mathrm{D}$ & $(3 / 2 / 2 / 2)$ & 512 & CERN \\
\hline "Honeycomb GEM" & double-mask & standard & $10 \times 10$ & $2 \mathrm{D}$ & $(3 / 2 / 2 / 2)$ & 512 & CERN \\
\hline "CMS Proto III" & single-mask & self-stretching & $10 \times 10$ & 1D & $(3 / 1 / 2 / 1)$ & 256 & CERN \\
\hline "CMS Proto IV" & single-mask & self-stretching & $30 \times 30$ & 1D & $(3 / 1 / 2 / 1)$ & 256 & CERN \\
\hline "Korean I" & double-mask & standard & $8 \times 8$ & 1D & $(3 / 2 / 2 / 2)$ & 256 & New Flex \\
\hline
\end{tabular}

self-stretching technique offers many advantages. No gluing nor soldering is required during the assembly procedure and the detector is produced without the need to place any spacers in the active area, in the gaps between the foils. The technique is very fast, for example this small prototype was assembled in only 1 hour. As an additional benefit, if needed, it allows for the detector to be re-opened in order to make modifications or repairs, or to replace a GEM foil.

The small "CMS Proto III" prototype was tested using an $\mathrm{x}$-ray gun with a $\mathrm{Cu}$ target in the RD51 lab. With an $\mathrm{Ar} / \mathrm{CO}_{2}$ $70 / 30$ gas mixture, the detector exhibited stable operation for a measured gain up to at least $3 \cdot 10^{4}$. The detector response was also observed to be very uniform across the GEM surface. Given these promising results, another prototype with dimensions $30 \times 30 \mathrm{~cm}^{2}$ was just recently (Sept. 2011) produced with the new technique. Initial tests of its performance are ongoing.

\section{Studies With LaRge Prototypes}

Besides the small prototypes described above, two full-size triple-GEM prototypes were produced, called "CMS Proto I" (April 2010) and "CMS Proto II" (March 2011). The design of the first prototype was reported earlier in [6]. The large prototypes have a trapezoidal shape with a GEM active area of $990 \times(220-455) \mathrm{mm}^{2}$. Their geometry follows the design that was foreseen for the RPCs to be installed in the first endcap disks in the high- $\eta$ region, ie. RE1/1 in Fig. 1. In fact, the GEM detectors are embedded in a similar aluminum cover box as was designed for the RPCs in that area. The chambers each cover a $10^{\circ}$ sector of the endcap disks. Fig. 3(a) shows an exploded view of "CMS Proto I", while Fig. 3(b) displays the general layout and gap size configuration of both detectors.

The GEM foils for both large-area prototypes were produced at CERN using a photolithographic process with a $50 \mu \mathrm{m}$ thick kapton sheet with a $5 \mu \mathrm{m}$ copper cladding on both sides. Given the size of these large prototypes, the singlemask technique had to be used to produce the foils. To limit the discharge probability, the foils are divided in 35 sectors of about $100 \mathrm{~cm}^{2}$ each. The drift electrodes, made of a $300 \mu \mathrm{m}$ kapton foil with a $5 \mu \mathrm{m}$ copper cladding, were glued directly to the $3 \mathrm{~mm}$ thick bottom aluminum plate of the detector cover boxes. The readout planes provide a one-dimensional readout with radial strips, i.e. pointing to the LHC beam line, with a pitch varying from 0.8 (short side) to $1.6 \mathrm{~mm}$ (long side) for "CMS Proto I" and from 0.6 to $1.2 \mathrm{~mm}$ for "CMS

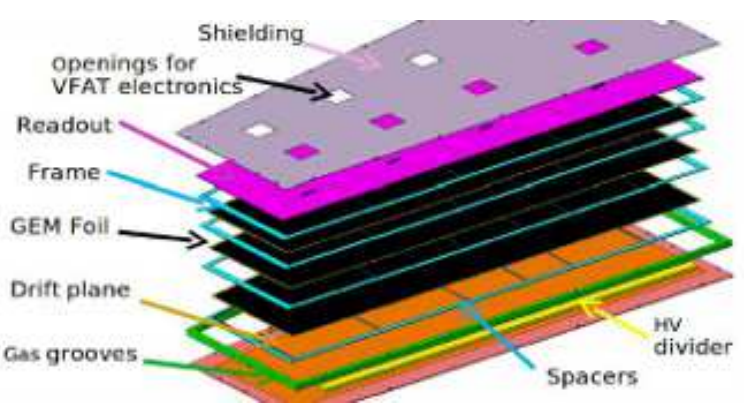

(a)

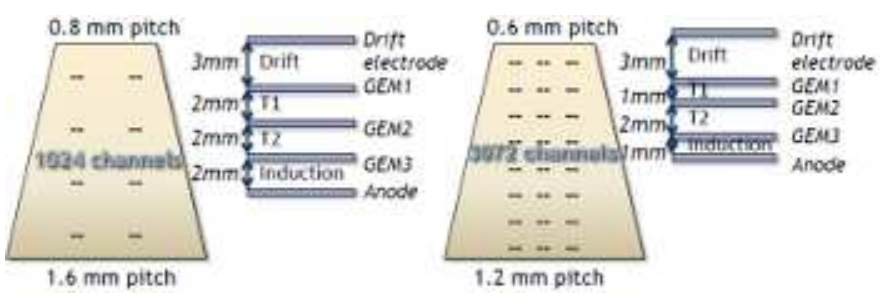

(b)

Fig. 3. (a) Exploded view of the first full-size triple-GEM prototype for CMS. (b) Layout and gap size configuration of both full-size prototypes.

Proto II". The first prototype was divided into four $\eta$-partitions with 256 strips per partition, while the second had eight partitions with 384 strips each. Following the results obtained with the small prototypes on the GEM timing performance, the gap size configuration for the second large prototype was $3 / 1 / 2 / 1 \mathrm{~mm}$ (drift, transf.1, transf.2, induction) in contrast to the $3 / 2 / 2 / 2 \mathrm{~mm}$ configuration for the first prototype. Different steps of the assembly procedure of the large prototypes are depicted in Fig. 4 Fig. 5 shows the gain curve for "CMS Proto I", measured with an x-ray gun in the RD51 lab.

The large prototypes were tested in $150 \mathrm{GeV}$ pion/muon beams at the CERN SPS $\mathrm{H} 2$ and $\mathrm{H} 4$ beamlines during several campaigns in October 2010, and from June to September 2011. Fig. 6(a) shows the first large-area prototype installed at the SPS H4 beam line in October 2010. A special mounting frame allowed to move the detector in the transverse plane with respect to the beamline, such that the beam could be pointed at different locations along the GEM surface in order to study the uniformity of the detector response. The influence of a magnetic field on the performance of the prototypes was also tested by inserting them in the M1 magnet at the SPS H2 beamline. This magnet can produce fields up to $3 \mathrm{~T}$. Inside 


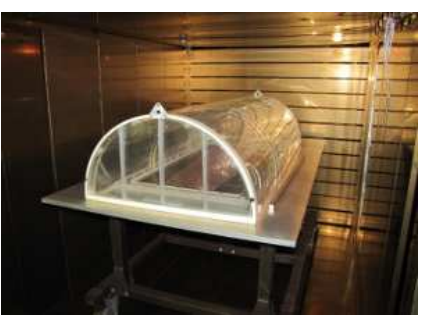

(a)

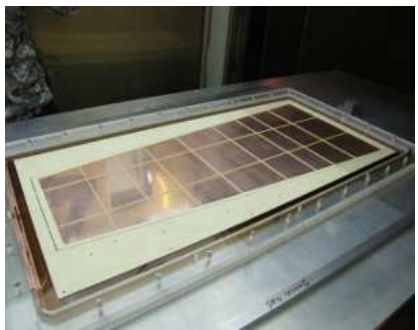

(c)

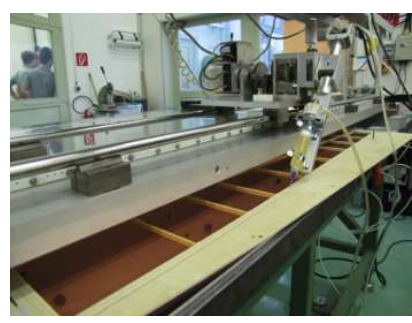

(b)

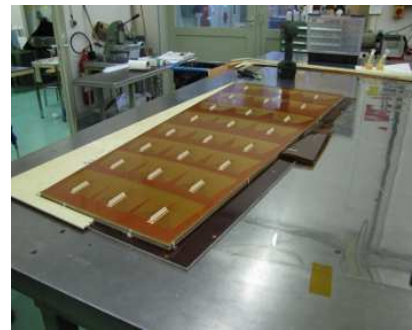

(d)
Fig. 4. Assembly procedure of the full-size CMS prototypes : (a) foil stretching in the oven; (b) gluing the spacer frames; (c) curing the glue; (d) mounting the readout plane.

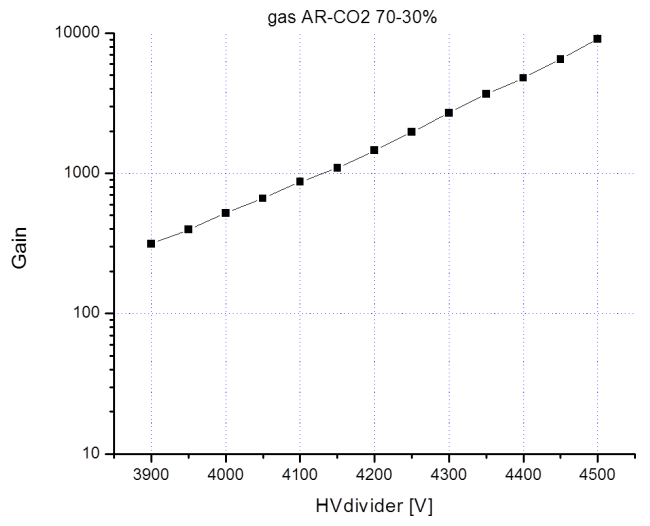

Fig. 5. Gain curve for the "CMS Proto I" measured with x-rays in the RD51 lab.

the CMS detector, the GEMs would be installed at a location where the magnetic field can reach up to $1.5 \mathrm{~T}$ with an angle between the magnetic field and the GEM electric field less than $8^{\circ}$.

Fig. 7 shows a few results obtained with "CMS Proto I" in the October 2010 campaign. Using an $\mathrm{Ar} / \mathrm{CO}_{2} 70 / 30$ gas mixture, with the beam pointing at the detector $\eta$-sector with the smallest average pitch size, ie. $1.05 \mathrm{~mm}$, a spatial resolution near the value expected from the strip pitch $(1050 / \sqrt{12}=305 \mu \mathrm{m})$, and a detector efficiency above $98 \%$ were obtained. The uniformity of the detector response across the GEM active area was found to be very good.

In Fig. 8 some results are shown for the "CMS Proto II" detector, which has a smaller strip pitch than "CMS Proto I", obtained during the August 2011 test beam campaign. With the detector flowing an $\mathrm{Ar} / \mathrm{CO}_{2} / \mathrm{CF}_{4} 45 / 15 / 40$ gas mixture, the data were taken with the $\mathrm{H} 4$ pion beam pointing at the region with the smallest strip pitch, i.e. $573 \mu \mathrm{m}$. An average cluster size of about 3 strips was found and for the space

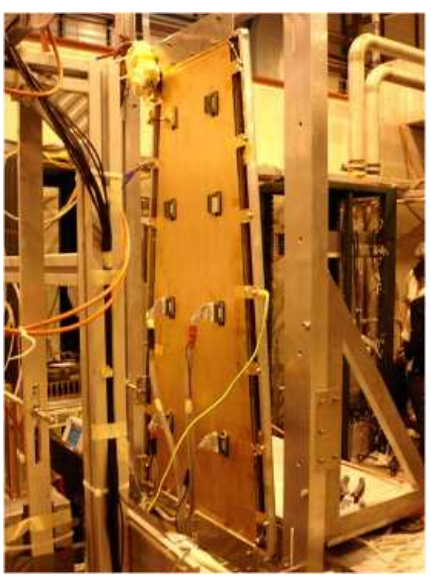

(a)

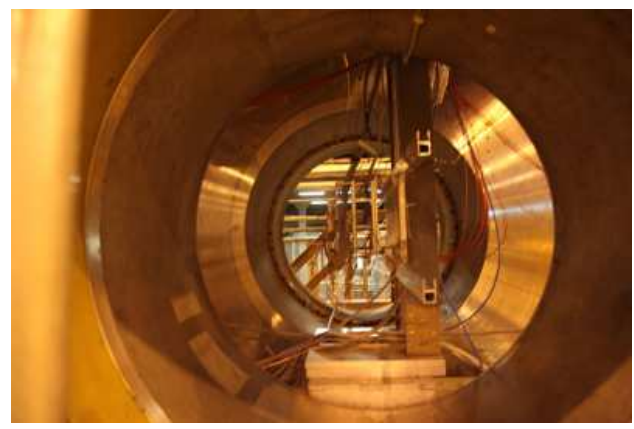

(b)

Fig. 6. (a) The "CMS Proto I" detector installed at the SPS H4 beam line (b) The "CMS Proto II" detector installed inside the CMS M1 magnet at the SPS $\mathrm{H} 2$ beam line.

resolution an upper limit of $103 \mu \mathrm{m}$ could be derived using the information on the charge sharing among adjacent strips, which is significantly better than the resolution expected from a purely digital readout (as used for the result in Fig. 7(a)), i.e. strip pitch $/ \sqrt{12}=573 / \sqrt{12}=165 \mu \mathrm{m}$.

In Fig. 9 the measured shift in the average strip cluster position due to a magnetic field is shown for "CMS Proto II" inside the M1 magnet. Measurements were performed in June and July 2011 using the SPS H2 $150 \mathrm{GeV}$ muon beam, with the detector flowing an $\mathrm{Ar} / \mathrm{CO}_{2} / \mathrm{CF}_{4} 45 / 15 / 40$ gas mixture. The angle between the GEM electric field and the external magnetic field could be varied by rotating the detector inside the M1 magnet. The data are in good agreement with a Garfield/Magboltz [7] simulation performed for a $90^{\circ}$ angle between the magnetic field and the GEM electric field. The measurement at $1.5 \mathrm{~T}$ was performed for an angle of $30^{\circ}$, which explains the observed small deviation between data and simulation for that point.

Up until recently, the readout of both the small and large prototypes was based on the TURBO front-end electronics with the VFAT [8] chip that produces digital output for its 128 analogue input channels. Efforts are ongoing to design a new, enhanced version of the VFAT2 chip to replace the present system. Furthermore, in the August-September 2011 campaigns the prototypes were for the first time successfully operated with the APV25 chip in combination with the Scal- 


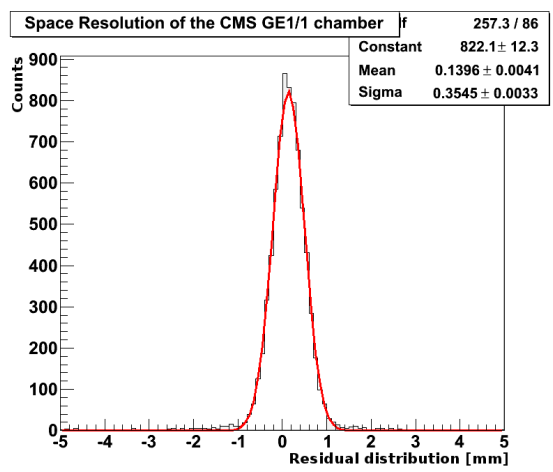

(a)

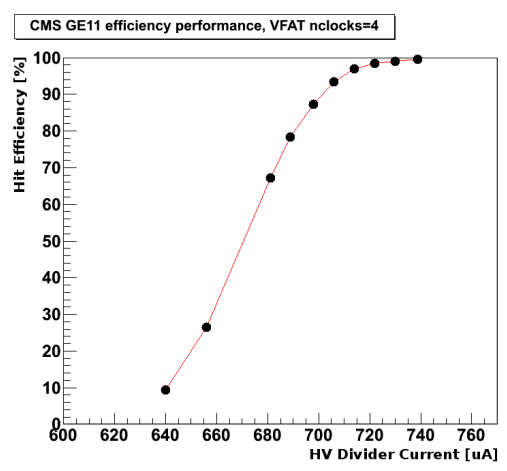

(b)

Fig. 7. Results obtained with "CMS Proto I" during the Oct. 2011 test beam campaign : (a) residual distribution; (b) detector hit efficiency as function of the current in the HV divider (proportional to the electric fields in the GEM).

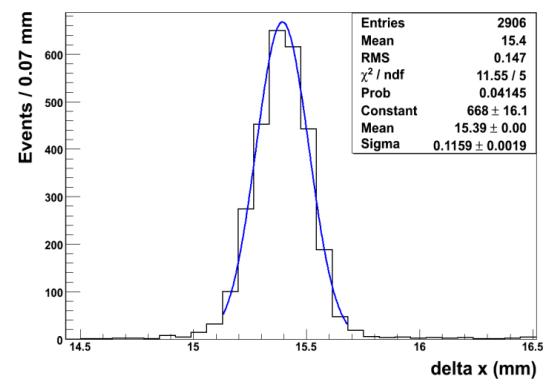

(a)

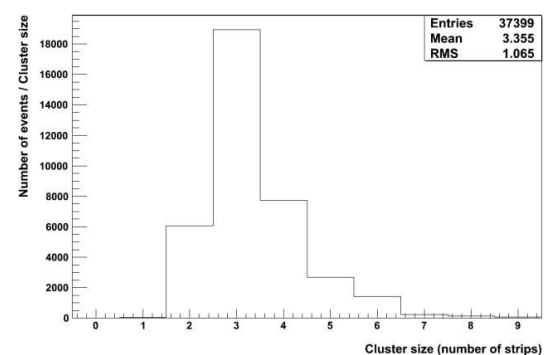

(b)

Fig. 8. Results obtained with "CMS Proto II" during the Aug. 2011 test beam campaign : (a) difference $\Delta x$ between hit positions measured with "CMS Proto II" and with a reference tracker GEM; (b) strip cluster size distribution.

able Readout System (SRS) [9] that is being developed by the RD51 Collaboration. The results shown in Fig. 8 were obtained with this system.

More details on test beam results obtained with the two

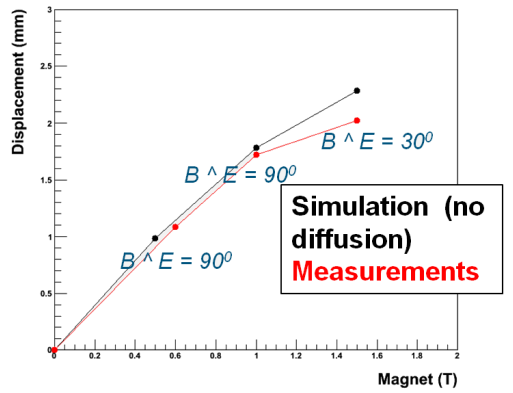

Fig. 9. Comparison of measured and simulated strip cluster position displacement in "CMS Proto II" due to an external magnetic field.

large-area prototypes can be found in [10].

\section{GEM PRODUCTION FACILITIES}

One of the possible scenarios that is presently under investigation for the installation of GEMs in the CMS detector, proposes the use of double chambers in each endcap disk, i.e. two chambers installed face-to-face. This would double the total number of detection layers and would thus greatly improve the tracking capability of the system. In such a scenario, to equip the $1.6<|\eta|<2.4$ region of the first and second CMS endcap disks, ie. the disks closest to the beam interaction point on either side, a total GEM foil area of order $500 \mathrm{~m}^{2}$ would be needed. In such a case, an industrialized production process becomes imperative.

With the ever-increasing demand for GEMs at CERN, the production facility will be significantly expanded in 20112013. A completely new production line with machines suitable for large numbers of large-area GEMs, up to about $2 \times 0.6 \mathrm{~m}^{2}$, is presently being put into place. In the end, the new facility will be housed in a dedicated building (\#107) at CERN. With this new setup, it is expected that the production cost of large-area GEMs could be lowered to about $1 \mathrm{kCHF} / \mathrm{GEM}$, with a production rate of $240 \mathrm{GEMs} /$ year.

Recently, an alternative GEM production facility was put in place at the New Flex company near Seoul in South Korea. Initial contacts with this company were made by CERN in November 2008 and in June 2011 a full technology transfer from CERN to New Flex was organized. In the meantime, the company has produced its very first $8 \times 8 \mathrm{~cm}^{2}$ double-mask triple-GEM, complete with voltage divider. The "Korean I" prototype (see Table \) was tested with a $\mathrm{Cu}$ target x-ray gun in the RD51 lab. The detector and voltage divider exhibited very stable operation with an $\mathrm{Ar} / \mathrm{CO}_{2} \quad 70 / 30$ gas mixture; smooth exponential gain curves were measured, similar to the CERN GEMs. The company is now progressing towards the production of large-area GEMs.

\section{Simulation Studies}

The CMS Monte Carlo simulation package is used to study and quantify the improvements in the trigger and muon reconstruction performance of an extended muon system in the CMS forward region as proposed here. As a starting point the geometry of the RPCs as described in the CMS Muon Technical Design Report [11] is taken $(T D R)$. To 


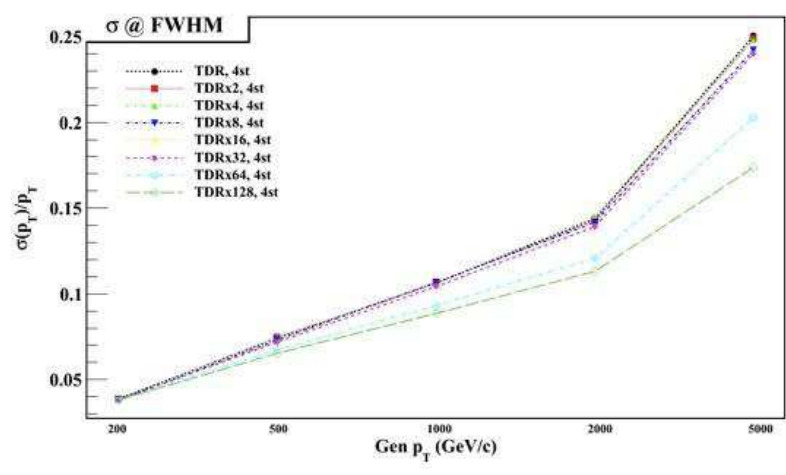

(a)

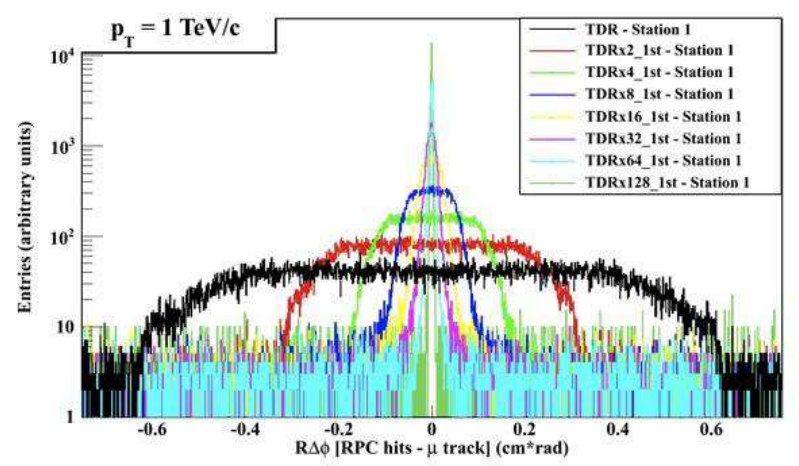

(b)

Fig. 10. Monte Carlo simulations for various detector granularities of the extended CMS muon endcap system : (a) muon momentum resolutions; (b) simulated spatial residual distributions for $p_{T}=1 \mathrm{TeV}$ muons.

mimic the GEM spatial resolution, the strip widths of the RPCs in the forward region $1.6<|\eta|<2.1$ are gradually increased $(n \times T D R)$ for the first two endcap stations. Using single-muon Monte Carlo simulation samples the effect of the GEM spatial resolution on the muon reconstruction can be studied. Examples of first results on the reconstructed muon momentum resolution and track residuals are shown in Fig. 10 for various simulated GEM spatial resolutions. For a $p_{T}=1 \mathrm{TeV}$ muon, up to a strip segmentation a factor 8 higher than in the current RPCs, the tracking resolution is dominated by the strip width; for even smaller strip widths the residual distribution becomes gaussian. These initial simulations are now being extended to study the improvements in the CMS muon trigger system. The effect of the extended muon system on the detector acceptance for various physics channels under study at CMS is also being addressed.

Simulations of the GEM detectors themselves are also being pursued [12]. The modeling of the GEM is based on ANSYS, while the Garfield/Magboltz packages are used to simulate the electron avalanches and interactions with the material. With these simulations the GEM gain is studied, and possible influences on the gain from various construction parameters, such as the outer hole diameter (see Fig. 11(a), the drift and induction field, the thickness of the metal layers, and also the Penning effect for the $\mathrm{Ar} / \mathrm{CO}_{2}$ gas mixture are addressed. Detailed studies of the electron avalanches also allow to study

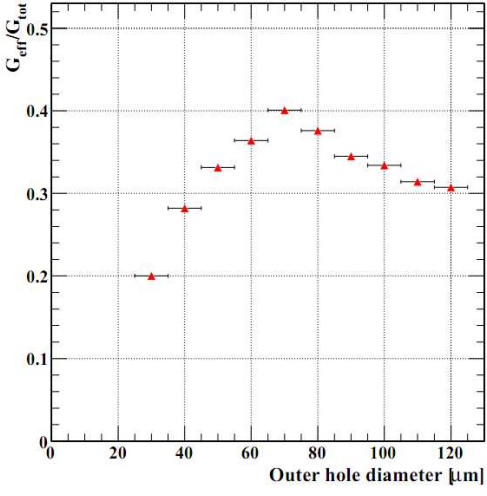

(a)

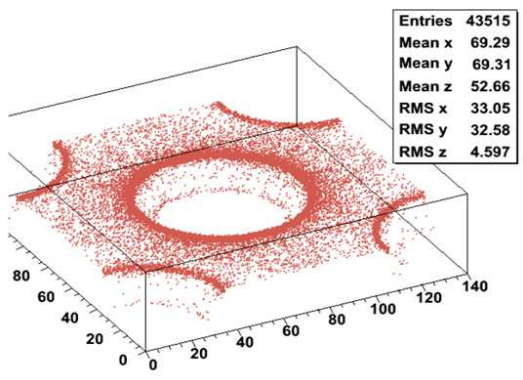

(b)

Fig. 11. Example results from GEM simulation studies : (a) effect of the outer hole diameter on the GEM gain; (b) locations of avalanche electron losses in the GEM material near a GEM hole.

electron losses due to the GEM geometry or attachment to gas molecules. As an example, Fig. 11(b) shows the position coordinates of electrons lost in the GEM material. Finally, the effect of an external magnetic field on the GEM performance is also studied in detail.

\section{SUMMARY AND OUTLOOK}

After intense studies on small-size prototypes, two fully operational large-area triple-GEM detectors were designed and assembled during 2010-2011. With several test beam campaigns at the CERN SPS H2 and H4 muon/pion beam lines it was demonstrated that these $990 \times(445-220) \mathrm{mm}^{2}$ prototypes exhibit stable and reliable operation and can be run with high gain and detector efficiency. The prototypes were tested inside a $1.5 \mathrm{~T}$ magnetic field, and could also be operated successfully with the RD51 Scalable Readout System. GEM detectors based on these full-size prototypes are being proposed for the extension of the CMS muon endcap system in the forward region. Simulation studies of the improved CMS trigger and physics performance with this system are ongoing.

\section{ACKNOWLEDGMENT}

This work has partly been performed in the framework of the RD51 Collaboration. The authors that are members of the CMS Collaboration would like to explicitly thank the RD51 Collaboration for its continuous support of this work, for the 
many fruitful discussions, for the close collaboration during the test beam campaigns and for dedicating part of their lab space at CERN to this project.

\section{REFERENCES}

[1] S. Chatrchyan et al., The CMS Collaboration, The CMS experiment at the CERN LHC, J. Instrum. 3 (2008) S08004

[2] The CMS Collaboration, Technical Proposal for the Upgrade of the CMS detector through 2020, CERN-LHCC-2011-006; CMS-UG-TP-1; LHCC$\mathrm{P}-004$

[3] The RD51 Collaboration, http://rd51-public.web.cern.ch/RD51-Public/

[4] D. Abbaneo et al., Characterization of GEM Detectors for Application in the CMS Muon Detection System, 2010 IEEE Nucl. Sci. Symp. Conf. Rec 1416-1422; RD51 Note 2010-005; arXiv:1012.3675 1 [physics.ins-det]

[5] S. Duarte Pinto et al., JINST 4 (2009) P12009; M. Villa et al., Nucl. Instr. and Meth. A628 (2011) 182-186

[6] D. Abbaneo et al., Construction of the first full-size GEM-based Prototype for the CMS High- $\eta$ Muon System, 2010 IEEE Nucl. Sci. Symp. Conf. Rec. 1909-1913; RD51 Note 2010-008; arXiv:1012.1524v2 [physics.insdet]

[7] R. Veenhof, Garfield - simulation of gaseous detectors, http://garfield.web.cern.ch/garfield/. S. Biagi, Magboltz - transport of electrons in gas mixtures, http://consult.cern.ch/writeups/magboltz/

[8] P. Aspell et al., VFAT2 : A front-end system on chip providing fast trigger information, digitized data storage and formatting for the charge sensitive readout of multi-channel silicon and gas particle detectors, Proc. of the Topical Workhop on Electronics for Particle Physic (TWEPP2007), Prague, Czech Republic, September 3-7, 2007

[9] S. Martoiu et al., Front-End Electronics for the Scalable Readout System of RD51, Contribution N43-5 to this conference

[10] D. Abbaneo et al., Test Beam Results of the GE1/1 Prototype for CMS High-Eta Muon System Future Upgrade, Contribution NP5.S-221 to this conference

[11] The CMS Collaboration, The Muon Project, CMS Technical Design Report, CERN/LHCC 97-32; CMS-TDR-003

[12] T. Moulik, Simulation of a Triple-GEM detector for a potential CMS muon tracking and trigger upgrade, Submitted to the Proc. of the Technology and Instrumentation in Particle Physics 2011 (TIPP 2011) Conference, 9-14 June, 2011, Chicago, IL, USA 